\title{
Establishment and Histopathological Study of Patient-derived Xenograft Models and Primary Cell Lines of Epithelioid Malignant Peritoneal Mesothelioma
}

\section{Zhi-Ran Yang}

Capital Medical University Affiliated Beijing Shijitan Hospital

Zhi-Gao Chen

Thorgene

Zhong-He Ji

Capital Medical University Affiliated Beijing Shijitan Hospital

Yu-Lin Lin

Capital Medical University Affiliated Beijing Shijitan Hospital Jue Zhang

Capital Medical University Affiliated Beijing Shijitan Hospital

Ru Ma

Capital Medical University Affiliated Beijing Shijitan Hospital

Zhao Li

Capital Medical University Affiliated Beijing Shijitan Hospital

Xi Jiang

Capital Medical University Affiliated Beijing Shijitan Hospital

Qian Chen

Thorgene

\section{Xue-Mei Du}

Capital Medical University Affiliated Beijing Shijitan Hospital

Yan Li ( $\square$ liyansd2@163.com )

Department of Peritoneal Cancer Surgery, Beijing Shijitan Hospital, Capital Medical University

\section{Research}

Keywords: Malignant peritoneal mesothelioma, PDX model, Primary cell lines, Histopathological study, WES

Posted Date: July 23rd, 2020 
DOI: https://doi.org/10.21203/rs.3.rs-45393/v1

License: (c) (1) This work is licensed under a Creative Commons Attribution 4.0 International License. Read Full License

Version of Record: A version of this preprint was published at Experimental Animals on January $1 \mathrm{st}$, 2021. See the published version at https://doi.org/10.1538/expanim.20-0119. 


\section{Abstract}

Background: Malignant peritoneal mesothelioma (MPM) is a rare malignancy with few effective therapies. This study established patient-derived xenograft (PDX) models and primary cell lines to provide a study platform for MPM in vitro and in vivo, and conducted histopathological analysis.

Methods: Human MPM surgical specimen was collected to establish PDX models and primary cell lines. Experimental peritoneal cancer index (ePCl) score was used to evaluate gross pathology, and histopathological study was conducted by hematoxylin-eosin (HE) and immunohistochemistry (IHC) staining. Pathological characteristics of the established primary MPM cell lines were analyzed by SwissGimsa and immunofluorescence (IF) staining. The whole-exome sequencing (WES) was performed to identify the mutant genes between models and the patient.

Results: MPM PDX models and primary cell lines were successfully established, replicated the pathological features of the patient. ePCl score of the female and male nude mice were $8.80 \pm 1.75$ and $9.20 \pm 1.81$ ( $P=0.6219)$, respectively. The HE staining showed that the tumor was epithelioid mesothelioma and invaded multiple organs. IHC staining showed that Calretinin, Cytokeratin 5/6, WT-1 and $\mathrm{Ki}-67$ were all positive. The Swiss-Gimsa and IF staining of primary cell lines were also consistent with the pathological characteristics of mesothelioma. The WES showed that 21 mutant genes were shared between models and the patient, and the genes related to tumorigenesis and development including BAP1, NF2, MTBP, NECTIN2, CDC23, LRPPRC, TRIM25, and DHRS2.

Conclusions: PDX models and primary cell lines of MPM were successfully established with the epithelioid mesothelioma identity confirmed by histopathological evidence. Moreover, our study has also illustrated the shared genomic profile between models and the patient, then potentially provided new targets for MPM treatment.

\section{Background}

Malignant peritoneal mesothelioma (MPM) is a rare malignancy characterized by highly malignant behavior and poor prognosis. MPM accounts for $10-30 \%$ of all malignant mesothelioma, and the median survival time of patient ranged from 5 to 12 months [1, 2]. The Peritoneal Surface Oncology Group International (PSOGI) recommends the combination of cytoreductive surgery (CRS) with hyperthermic intraperitoneal chemotherapy (HIPEC) as the standard treatment [3, 4]. Although CRS + HIPEC could extend the median survival time of patients to roughly 3 years, it proves little efficacy for some patients [5-7]. Therefore, it is urgent and necessary to explore novel effective treatments to reverse such dismal trend.

Animal models are important platforms for studying disease mechanisms and exploring new therapeutic strategies. Currently, there are mainly three types animal models of malignant mesothelioma, which including spontaneous models [8, 9], induced models [10], and transplanted models [11, 12]. The spontaneous models and induced models are the most similar to humans in terms of tumor occurrence, 
they reflect the susceptibility of animal tumors and the degree of aggregation of environmental carcinogens, and may help to discover environmental carcinogens. However, the disadvantages of these models limited its application, such as the different course of disease, the longer induction time, the lower induction rate, and unsuitable for drug screening. To the opposite, the transplanted models are characterized by the lower induction time, the higher induction rate, and suitable for conducting drug intervention experiments, thus the xenograft models are currently the best tool for studying human MPM. Up to now, however, there has been few xenograft models of human MPM from China. Therefore, it is requisite to establish patient-derived xenograft (PDX) models of MPM to further explore the mechanisms, discover new targets, and conduct drug screening study.

This study directly used MPM surgical specimens to establish MPM PDX models in nude mice, and primary cell lines. The major pathological features were also systematically studied.

\section{Materials And Methods}

\subsection{Patient and tumor sample}

Tumor samples were obtained from a 63-year-old female patient who complained of persistent abdominal pain for 4 months. The patient received CRS + HIPEC and was diagnosed as epithelial malignant peritoneal mesothelioma. Resected tumors were used for the establishment of PDX models. Informed consent has been obtained from the patient, and all experiments were performed under the guideline of the Declaration of Helsinki and Institutional Animal Care and Use Committee Health guidelines (IACUC20190306).

\subsection{Animals}

Specific pathogen free BALB/c nu/nu mice, 4-5 weeks old, 16-18 g, were purchased from Beijing Vital River Laboratory Animal Technology Co., Ltd. (Beijing, China) and were raised individually in ventilated cages in a barrier environment at the temperature of $20-26^{\circ} \mathrm{C}$, in the humidity of $40-70 \%$ at Beijing Percans Oncology (Beijing, China). After a quarantine period of 7 days, mice were prepared for model establishment. The whole study procedures were illustrated in Fig. 1.

\subsection{Establishment of MPM PDX models}

Tumor specimens were cut into pieces sized of $1 \mathrm{~mm}^{3}$, and then washed with RPMI 1640 medium (Corning, New York, USA) and inoculated subcutaneously on back of nude mice using a $25 \mathrm{G}$ trocar sheath needle. The volume of subcutaneous tumors was measured every three days. When it reached $500 \mathrm{~mm}^{3}$, the tumors were resected for characterization and passaging. The resected tumors were divided into four groups, including (1) histopathological study, (2) model establishment, (3) primary cell, and (4) subcutaneous inoculation on back of nude mice for passage. Subcutaneous models were reestablished according to the same protocol. Two nude mice were inoculated each time. 
After 4 passages, subcutaneous (s.c.) tumor was cut into pieces and homogenized in a glass tissue homogenizer containing $1.5 \mathrm{ml}$ RPMI 1640 medium to produce $2.5 \mathrm{ml}$ tumor cell homogenate. 20 nude mice (10 males and 10 females) were used for MPM PDX model establishment. Each nude mouse was inoculated with $100 \mu \mathrm{L}$ of tumor cell homogenate in the left lower abdominal cavity using a $25 \mathrm{G}$ trocar sheath needle. Mice was closely monitored daily, with the body weight measured every there days.

\subsection{Gross pathological study}

Mice were sacrificed for autopsy on D14 of grafting. Tumor growth and progression features were recorded. Experimental peritoneal cancer index $(\mathrm{ePCl})$ was used to evaluate the extent of tumor dissemination, based on published studies by Shao [13]. The abdominal-pelvic cavity was divided into 4 subareas, and lesion size score (LS) in each subarea is determined by the diameter of the largest tumor: LS-0, no visible tumor; LS-1, diameter $\leq 0.2 \mathrm{~cm}$; LS-2, $0.2 \mathrm{~cm}<$ diameter $\leq 0.5 \mathrm{~cm}$; LS-3, diameter $>0.5 \mathrm{~cm}$; Malignant ascites, 1 point. The accumulative ePCl score ranges from 0 to 13 (Fig. 2A).

\subsection{Histopathological study}

Tumor and organ tissues were fixed in $4 \%$ formaldehyde solution for $48 \mathrm{~h}$, followed by routine dehydration, paraffin embedding and section $(4 \mu \mathrm{m})$. Hematoxylin-eosin (HE) staining (Dako Hematoxylin,Dako Eosin and Dako Bluing Buffer, catalog number CS701) was performed on Dako CoverStainer (Agilent Technologies, Inc., California, USA). Immunohistochemistry (IHC) staining was performed on intelliPATH FLX (BIOCARE MEDICAL, LLC, California, USA) with Polymer Immunohistochemical Detection System (Wuxi OriGene Technologies, Inc., Wuxi, China, catalog number MA-2000). Primary antibodies, including anti-Calretinin, -Cytokeratin 5/6, -WT-1 and -Ki-67, were used for IHC analysis (Table 1). The images were captured using Zeiss Axio Scope.A1 and Mshot Microscopic Camera MS60. All HE and IHC sections were reviewed by 3 senior pathologists. 
Table 1

Primary antibodies used for immunohistochemistry

\begin{tabular}{|llll|}
\hline Primary antibodies & Supplier & $\begin{array}{l}\text { Catalog } \\
\text { number }\end{array}$ & Dilution \\
\hline rabbit anti-human Calretinin antibody & $\begin{array}{l}\text { Zhongshan Golden } \\
\text { Bridge }\end{array}$ & Poly & Ready to use \\
\hline mouse anti-human WT-1 antibody & Genetic technology & $6 \mathrm{~F}-\mathrm{H} 2$ & Ready to use \\
\hline $\begin{array}{l}\text { mouse anti-human Cytokeratin 5/6 } \\
\text { antibody }\end{array}$ & OriGene & OTI1C7 & Ready to use \\
\hline \begin{tabular}{l} 
mouse anti-human Ki-67 antibody \\
\hline
\end{tabular} & OriGene & UMAB107 & $1: 100$ \\
\hline
\end{tabular}

Table 2. Gene mutations related to tumor shared between the models and the patient

\begin{tabular}{llllll} 
Gene & Mutation Abundance (\%) & $\begin{array}{l}\text { Type of } \\
\text { mutation }\end{array}$ & Exon & $\begin{array}{l}\text { Base } \\
\text { mutation }\end{array}$ & $\begin{array}{l}\text { Amino acids } \\
\text { mutation }\end{array}$ \\
\hline BAP1 & 99.9 & FM & 13 & c.1464delC & p.P488fs \\
\hline NF2 & 90.0 & FM & 5 & c.484delT & p.F162fs \\
\hline MTBP & 60.3 & FM & 2 & c.144delC & p.H48fs \\
\hline NECTIN2 & 56.6 & SNV & 3 & c.619A $>$ T & p.T207S \\
\hline CDC23 & 48.9 & SNV & 13 & c.1421C > G & p.A474G \\
\hline LRPPRC & 47.2 & FM & 20 & c.1978dupA & p.T660fs \\
\hline TRIM25 & 33.7 & SNV & 4 & c.998T >C & pL333P \\
\hline DHRS2 & 32.1 & SNV & 8 & c.722G $>$ A & p.R241K \\
\hline
\end{tabular}

Abbreviations: FM, frameshift mutation; SNV, single nucleotide mutation.

\subsection{Establishment of MPM primary cell lines}

Isolation and purification

The s.c. tumor was cut into $1 \mathrm{~mm}^{3}$ pieces, which were then transferred to a centrifuge tube containing dispersase II (Sigma, USA) and digested on $37^{\circ} \mathrm{C}$ incubator for $1 \mathrm{~h}$. The cell suspension was filtered through a $70 \mu \mathrm{m}$ cell filter into a $50 \mathrm{ml}$ centrifuge tube, and was then centrifuged at $515 \mathrm{~g}$ for $5 \mathrm{~min}$, followed by re-suspending in culture medium for culturing.

After 5 days of culturing, dispersase II was added and incubated at $37^{\circ} \mathrm{C}$ for $10 \mathrm{~min}$. Most of the tumor cells were detached from the flask bottom, while the fibroblasts were still adherent to the flask. The detached tumor cells were centrifuged at $515 \mathrm{~g}$ for $5 \mathrm{~min}$ and were re-suspended in culture medium for culturing. 
Cell culture

Cells were cultured in DMEM containing 10\% fetal bovine serum (FBS; Gibco, USA), 100 U/mL penicillin and $100 \mu \mathrm{g} / \mathrm{mL}$ streptomycin (penicillin-streptomycin-glutamine; Gibco, USA) at $37^{\circ} \mathrm{C}, 5 \% \mathrm{CO}_{2}$ in a humidified Forma Steri-Cycle $\mathrm{CO}_{2}$ Incubator (Thermo Fisher Scientific, Massachusetts, USA).

\subsection{Cell identification}

Swiss-Gimsa staining

Sterile round slides were added onto the 24-well plate, and $200 \mu$ l culture medium was added into each well. Cells were diluted to the concentration of $1 \times 10^{6}$ cells $/ \mathrm{ml}$, and $30 \mu \mathrm{l}$ of cell suspension was added to each well. After 24 hours of incubation, cells were fixed by using ice-cold methanol for 3 min and were stained with Swiss-Gimsa (Solarbio, China) for $5 \mathrm{~min}$. The morphology of cells was observed under an EVOS $^{\mathrm{TM}}$ XL Core Configured Microscope (Thermo Fisher Scientific, Waltham, MA, USA).

Immunofluorescence staining

Cells were added to 24-well plate according to above method, fixed with ice-methanol for 5 min. Primary mouse anti-Calretinin monoclonal antibody (1F5H1, Abcam, UK; 1:200) and anti-Cytokeratin 5 monoclonal antibody (XM26, Abcam, UK; 1:250) were revealed with specific goat anti-mouse Alexa Fluorß 555 (IgG H\&L)-conjugated secondary antibodies (ab150114, Abcam, UK; 1:1000) for 30 min at $37^{\circ} \mathrm{C}$. Nuclei were stained with 4',6-diamidino-2-phenylindole (DAPI; Beyotime Institute of Biotechnology, China). Pictures were captured under a fluorescence microscope (Olympus, BX43, Japan).

\subsection{Study on the molecular pathology by whole-exome sequencing (WES)}

The WES sequencing protocol prescribed by Giannakis et al [14] was used in our study. In terms of models, the tumor tissues preserved in the nitrogen were centrifuged with $1 \mathrm{~min}$, the centrifugal rate of $10,000 \mathrm{r} / \mathrm{min}$. For the patient, the formalin-fixed, paraffin-embedded tumor tissues were sectioned into $5 \mu \mathrm{m}$ for sequencing, and the normal tissues adjacent to tumor were regarded as control. QIAGEN DNA kit (QIAGEN NV, Hilden, German) was used to extract genomic DNA, and the Quant-iT Pico Green dsDNA Assay Kit (Invitrogen/ThermoFisher SCIENTIFIC, Massachusetts, USA) was conducted to check the DNA quality. The whole-exome capture libraries (final concentration $>20 \mathrm{ng} / \mu \mathrm{L}$ ) were constructed by shearing, end repair, phosphorylation and ligation to barcoded sequencing adaptors. SureSelectXT Human All Exon V6 (Agilent Technologies, California, USA) was used to capture DNA and the Illumina X10 platform (Illumina Inc, California, USA) was then used to sequenced the samples.

The method of MuTect [15] was performed to detect the somatic mutations, and the somatic cell insertion and deletion markers were detected by the method of Indelocator and Strelka [16]. The mutation analysis of WES data was performed using human genome build hg19 as the reference genome.

\subsection{Statistical analysis}


Data are presented as mean \pm standard deviation (SD) when normal distribution is satisfied. Statistical analyses and image processing were performed using GraphPad Prism 8.0.1 (GraphPad, San Diego, CA, USA) and Adobe Photoshop 2017 CC software (Adobe Systems, San Jose, CA, USA). Student's t-test were applied to evaluated statistical significance. $P \otimes 0.05$ was considered statistically significant.

\section{Results}

\subsection{Establishment and pathological features of the new MPM PDX models}

General status

From the day of grafting to autopsy, the general conditions of nude mice, such as diet, excretion, mental state, were normal. The body weight increased steadily from D1 to D6, followed by a plateau from D6 to D10, and then decreased since D10 to D14. Female and male nude mice had the same body weight change trend (Fig. 2B).

Gross pathology

On the 14th day of grafting, the mice were sacrificed to observe the situation of model establishment. The rate of model establishment was $100 \%(20 / 20)$. The tumors were white, single or fused, and invaded multiple organs, including the mesentery, 95\% (19/20; Fig. 2C), diaphragm, 45\% (9/20; Fig. 2D), liver, 50\% (10/20), stomach, 40\% (8/20), pancreas, $10 \%$ (2/20), spleen, 65\% (13/20; Fig. 2E), kidney, $40 \%(8 / 20)$, and pelvic cavity, $100 \%(20 / 20 ;$ Fig. $2 \mathrm{~F})$. The ePCl score of female and male nude mice were $8.80 \pm 1.75$ and $9.20 \pm 1.81(P=0.6219 ;$ Fig. $2 \mathrm{G})$, respectively.

Histopathological study

The results of HE staining showed that the tumor tissue of nude mice was epithelioid mesothelioma. The tumor cells were morphologically diverse, large in size, markedly heteromorphic, and invaded multiple organs, such as diaphragm, liver (Fig. 3A-B), pancreas (Fig. 3C-D), spleen, kidney, mesentery (Fig. 3E-F), and uterus (Fig. 3G-H), which is consistent with that of the patient (Fig. $3 \mathrm{I}-\mathrm{L}$ ).

Immunohistochemical staining of the MPM PDX models showed that the Calretinin, Cytokeratin 5/6, WT1 and Ki-67 were all positive (Fig. 4A-D). These results were also consistent with the patient (Fig. 4E-H).

\subsection{Establishment and cytological characteristics of primary MPM cell lines}

Swiss-Gimsa staining 
The morphology of MPM cells under inverted phase contrast microscope was shown in Fig. 5A. SwissGimsa staining was performed to reveal the diverse cell morphology, such as varying size, shape, nucleus number, and the visibility of nucleolus (Fig. 5B).

Immunofluorescence staining

The immunofluorescence staining was performed to confirm the expression of Calretinin and Cytokeratin 5 in the established MPM cells (Fig. 5C), which was in accordance with the cytological characteristics of epithelioid mesothelioma.

\subsection{Potential molecular features of MPM PDX models}

26 and 36 mutant genes with higher frequency (mutation abundance $>10 \%$ ) were found in PDX models and the patient, respectively. 21 mutant genes were shared in the two groups, among them, the genes related to tumorigenesis and development including BAP1, NF2, MTBP, NECTIN2, CDC23, LRPPRC, TRIM25, and DHRS2 (Table 2.).

\section{Discussion}

By using surgical specimens collected from MPM patient, we established PDX models and primary cell lines of MPM, which highly replicated the pathological development of clinical patients. The biological behaviors of the PDX models were characterized by highly malignant degree and strong invasion and metastasis ability. Tumors invaded multiple organs, including diaphragm, liver, spleen, kidney, mesentery, and organs of pelvic cavity. The histopathological results also showed that the pathological features of the models and primary cell lines were epithelioid mesothelioma, consistent with that of the patient. Therefore, the established PDX models and primary cancer cell lines provided a platform for in vivo and in vitro studies of the pathological mechanism and the clinical intervention for MPM.

By observing the body weight change trend of nude mice during grafting, we found that the plateau was appeared on D6, and the body weight decreased on D10, which was not affected by gender. The body weight change suggested that the PDX tumors had an impact on the general state of nude mice on D6 of grafting, and the nude mice were in a consumption state on D10. Therefore, the body weight change during the grafting was of reference significance in judging the impact of tumors on the host and the biological behavior of the tumors. Moreover, the EPCT score of female and male nude mice also indicated that gender did not affect the establishment of models.

In terms of molecular pathology research, 21 genes with high mutation abundance were shared between the PDX models and the patient. The genes associated with tumorigenesis and development including BAP1, NF2, MTBP, NECTIN2, and so on. Both BAP1 and NF2 were frameshift mutations, with mutation abundances of $99.9 \%$ and $90.0 \%$, respectively. Evidence previously reported that mutations or deletions of BAP1 and NF2 were found in malignant mesothelioma, with the mutation abundances of $57 \%$ and $35 \%$, respectively, NF2 mutations or deletions were associated with poor prognosis, and provided a new target for MPM, while the relationship between the mutation of BAP1 and the prognosis in MPM patients was still controversial [17]. NECTIN2 was an adhesion molecule that played an important role in tumor growth 
and metastasis. In addition, NECTIN2 was also a ligand of NK cell activating receptor CD226 and highly expressed in colon cancer tissues. Upregulating the expression intensity of NECTIN2 in colon cancer tissues can activate the killing effect of NK cells $[18,19]$. However, the function of NECTIN2 mutation in MPM are unclear. Moreover, the mutant genes between the established models and the patient were not completely identical, 5 mutant genes were only existed in models, and 15 mutant genes in patient. The reasons are may as follows, (1) tumor heterogeneity led to the different mutant genes between the two groups, (2) the tumor tissues sequenced in the patient were paraffin section, while the models were fresh frozen tissues, which may led to the different results, and (3) the genes of the models may have changed due to the environment factors during the passaging process. However, the established PDX models still own the key genes and can conduct related studies.

Some animal models and few primary cell lines of MPM have been reported. Feng et al [20] inoculated mesothelioma cell lines $\mathrm{NCl}-\mathrm{H} 226$ into the abdominal cavity of 6-week-old female NCr-nu/nu nude mice to establish an MPM model, and conducted drug intervention study by using this model. The tumors of the model invaded the diaphragm, liver, pancreas, and peritoneum of nude mice. Yang et al [21] inoculated a AB12 cell line, a malignant pleural mesothelioma cell line, into 5-week-old female BALB/C nude mice to form a subcutaneous tumor model. They observed the changes in tumor volume and weight of this model to determine the efficacy of drugs. However, few primary cell lines of MPM have been reported. Compared with the existing MPM models, our established models had the following characteristics, (1) using MPM surgical specimens to directly grafted nude mice to establish the MPM PDX models, this method not only saved time and cost, but also simulated maximally the tumor biological behavior of the clinical patients, (2) the established PDX models exhibited the pathological features highly consistent with the clinicopathological characteristics of MPM in terms of gross pathology and histopathology, and (3) the PDX models own the key mutant genes for the development of MPM, which may become a research tool for exploring the mechanism and new therapeutic targets for MPM. In addition, we also cultured primary MPM cell lines derived from the established subcutaneous models, and thus provided a platform in vitro study.

In conclusion, we established PDX models and primary cell lines to recapitulate the biological characteristics of MPM and provided a novel and promising platform for in vivo and in vitro study on MPM.

\section{Abbreviations}

MPM, malignant peritoneal mesothelioma; PDX, patient-derived xenograft; ePCl, experimental peritoneal cancer index; PSOGI, peritoneal surface oncology group international; CRS, cytoreductive surgery; HIPEC, hyperthermic intraperitoneal chemotherapy; s.c., subcutaneous; HE, hematoxylin-eosin; IHC, immunohistochemistry; IF, immunofluorescence; WES, whole-exome sequencing; FM, frameshift mutation; SNV, single nucleotide mutation.

\section{Declarations}




\section{Acknowledgments}

Not applicable.

\section{Funding}

This study was supported by funding from Beijing Municipal Administration of Hospitals' Ascent Plan (DFL20180701); Special Fund for the Capital Characteristic Clinical Medicine Development Project (Z161100000516077); Beijing Municipal Grant for Medical Talents Group on Peritoneal Surface Oncology (2017400003235J007); Key Discipline Development Fund of Beijing Shijitan Hospital, Capital Medical University (2016fmzlwk); Beijing Natural Science Foundation (7172108); Beijing Health and Science Technology Achievement and Appropriate Technology Promotion Project (2018-TG-27).

\section{Competing interests}

The authors declare that they have no competing interests.

\section{Availability of data and materials}

All data and material generated or used during the study are available from the corresponding author by request.

\section{Authors' contributions}

YZR and CZG were involved in study design, research implementation and data interpretation, and manuscript writing. JZH, LYL, ZJ, MR, LZ, JX were involved in conduct and reporting of the work described in the article. CQ, DXM and LY were contributed to study design, data analysis and interpretation, design of figures. All authors were involved in manuscript development, did a full review of the article, have approved the final version of the manuscript, and are responsible for all content.

\section{Ethics approval and consent to participate}

All experiments involving animals were performed under the guideline of the Declaration of Helsinki and Institutional Animal Care and Use Committee Health guidelines (IACUC20190306).

\section{Consent for publication}

The samples used in the study and the publication have obtained the patient's consent. 


\section{References}

1. Price B. Analysis of current trends in United States mesothelioma incidence[J]. Am J Epidemiol. 1997, 145(3): 211-218.

2. Kaya $\mathrm{H}$, Sezgi $\mathrm{C}$, Tanrikulu AC, et al. Prognostic factors influencing survival in 35 patients with malignant peritoneal mesothelioma[J]. Neoplasma. 2014, 61(4): 433-438.

3. Feldman AL, Libutti SK, Pingpank JF, et al. Analysis of factors associated with outcome in patients with malignant peritoneal mesothelioma undergoing surgical debulking and intraperitoneal chemotherapy[J]. J Clin Oncol. 2003, 21(24): 4560-4567.

4. Sugarbaker PH, Yan TD, Stuart OA, et al. Comprehensive management of diffuse malignant peritoneal mesothelioma[J]. Eur J Surg Oncol. 2006, 32(6): 686-691.

5. Yano H, Moran BJ, Cecil TD, et al. Cytoreductive surgery and intraperitoneal chemotherapy for peritoneal mesothelioma[J]. Eur J Surg Oncol. 2009, 35(9): 980-985.

6. Hompes D, D'Hoore A, Van Cutsem E, et al. The treatment of peritoneal carcinomatosis of colorectal cancer with complete cytoreductive surgery and hyperthermic intraperitoneal peroperative chemotherapy (HIPEC) with oxaliplatin: a Belgian multicentre prospective phase II clinical study[J]. Ann Surg Oncol. 2012, 19(7): 2186-2194.

7. Bretcha-Boix P, Farré-Alegre J, Sureda M, et al. Cytoreductive surgery and perioperative intraperitoneal chemotherapy in patients with peritoneal carcinomatosis of colonic origin: outcomes after 7 years' experience of a new centre for peritoneal surface malignancies[J]. Clin TransI Oncol. 2010, 12(6): 437-442.

8. De Nardo P. Veterinary environmental epidemiology: the case of respiratory pathology in the dog[J]. Ann Ist Super Sanita. 1997, 33(4): 587-593.

9. Kim JH, Choi YK, Yoon HY, et al. Juvenile malignant mesothelioma in a dog[J]. J Vet Med Sci. 2002, 64(3): 269-271.

10. Wangner JC, Berry G. Mesotheliomas in rats following inoculation with asbestos[J]. $\mathrm{Br} \mathrm{J}$ Cancer. 1969, 23(3): 567-581.

11. Colt Hg, Astoul, Wang $X$, et al. Clinical course of human epithelial-type malignant pleural mesothelioma replicated in an orthotopictransplant nude mouse model[J]. Anticancer Res. 1996, 16(2): 633-639.

12. Astoul P, Boutin C. An experimental model of pleural cancer. Value of orthotopic implantation of human tumor tissue in nude mice[J]. Rev Mal Respir. 1997, 14(5): 355-362.

13. Shao LH, Liu SP, Hou JX, et al. Cathepsin B cleavable novel prodrug Ac-Phe-Lys-PABC-ADM enhances efficacy at reduced toxicity in treating gastric cancer peritoneal carcinomatosis: an experimental study[J]. Cancer. 2012, 118(11): 2986-2996.

14. Giannakis M, Mu X, Shukla S, et al. Genomic correlates of immune-cell infiltrates in colorectal carcinoma[J]. Cell Rep. 2016;17(4):1206. 
15. Cibulskis K, Lawrence MS, Carter SL, et al. Sensitive detection of somatic point mutations in impure and heterogeneous cancer samples[J]. Nat Biotechnol, 2013, 31(3): 213-219.

16. Saunders CT, Wong WS, Swamy S, et al. Strelka: accurate somatic small-variant calling from sequenced tumor-normal sample pairs[J]. Bioinformatics, 2012, 28(14): 1811-1817.

17. Singhi AD, Krasinskas AM, Choudry HA, et al. The prognostic significance of BAP1, NF2, and CDKN2A in malignant peritoneal mesothelioma[J]. Mod Pathol. 2016, 29(1): 14-24.

18. Bekes I, Lob S, Holzheu I, et al. Nectin-2 in ovarian cancer: How is it expressed and what might be its functional role? [J]. Cancer Sci. 2019, 110(6): 1872-1882.

19. Molfetta R, Milito ND, Zitti B, et al. The Ubiquitin-proteasome pathway regulates Nectin2/CD112 expression and impairs NK cell recognition and killing[J]. Eur J Immunol. 2019, 49(6): 873-883.

20. Feng MQ, Zhang JL, Anver M, et al. In vivo imaging of human malignant mesothelioma grown orthotopically in the peritoneal cavity of nude mice[J]. J Cancer, 2011, 2(1): 123-131.

21. Yang $H J$, Wang $Y$, Vino $T$, et al. Withaferin A inhibits the proteasome activity in mesothelioma in vitro and in vivo[J]. PLoS One, 2012, 7(8): e41214.

\section{Figures}

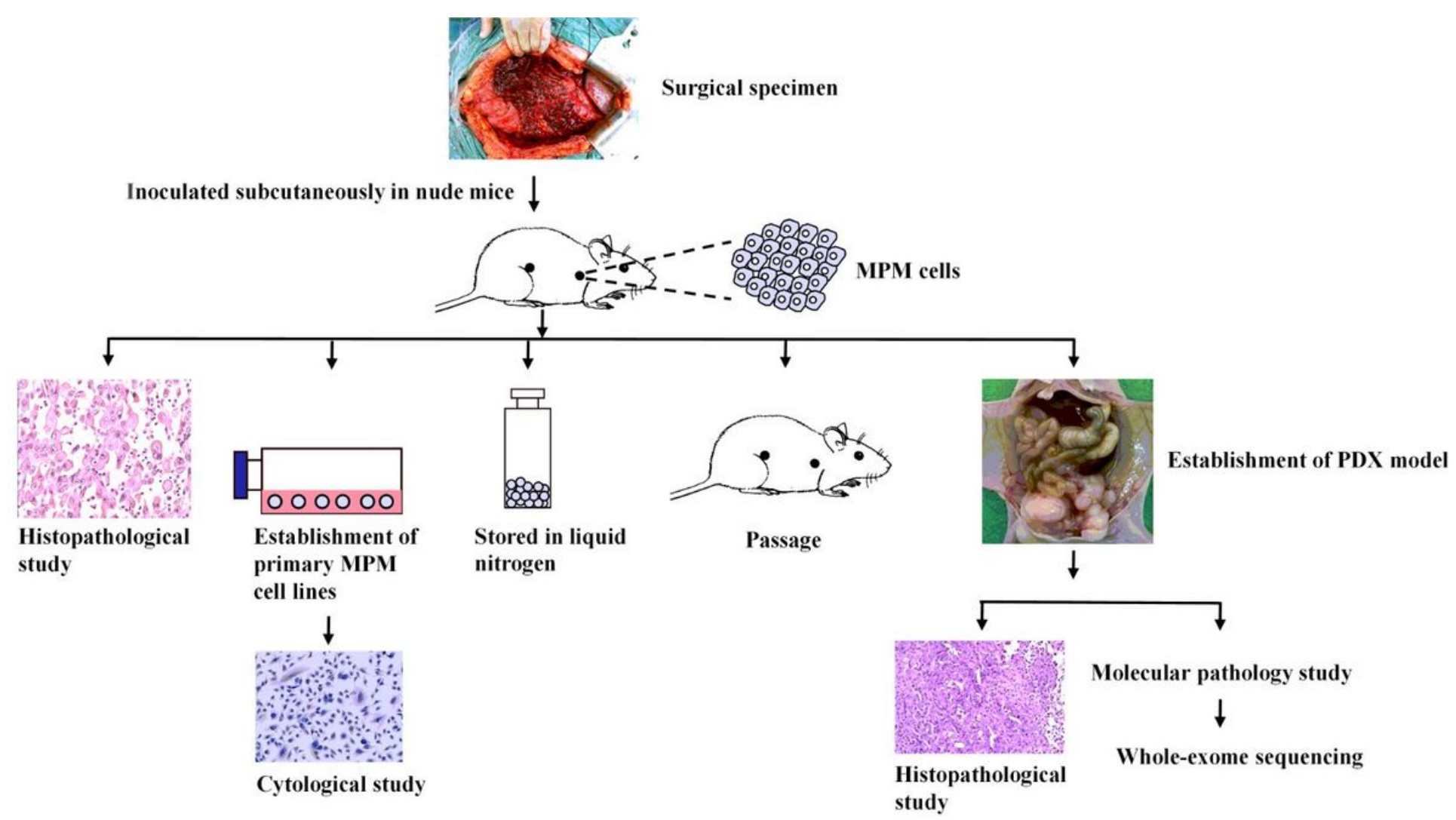

Figure 1

Experimental procedures and histopathological analysis of the established malignant peritoneal mesothelioma (MPM) patient-derived xenograft (PDX) models and primary cell lines. 


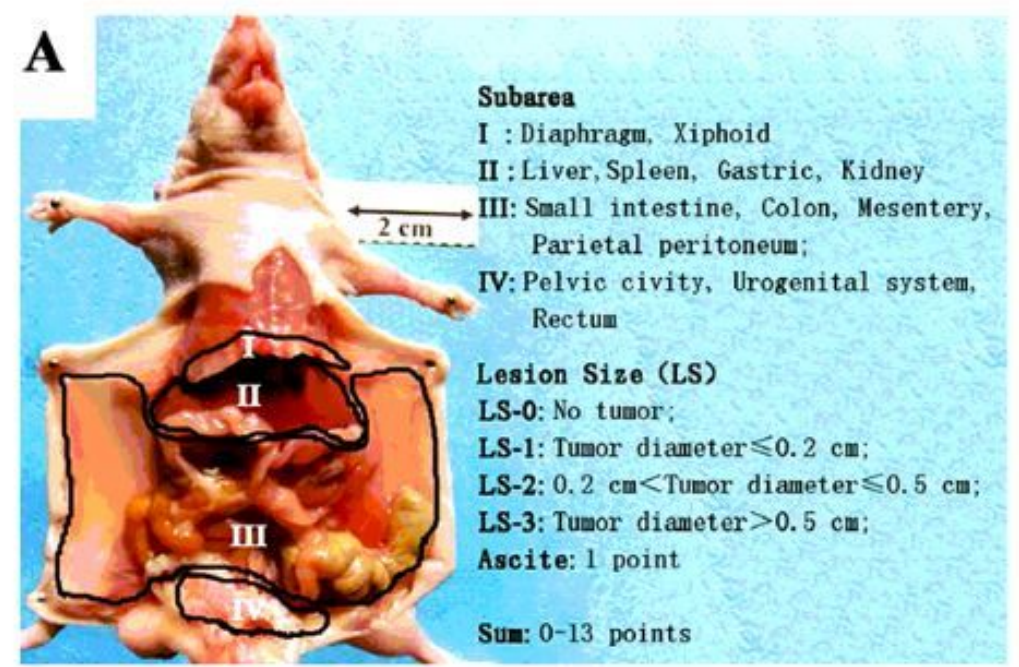

\section{B}
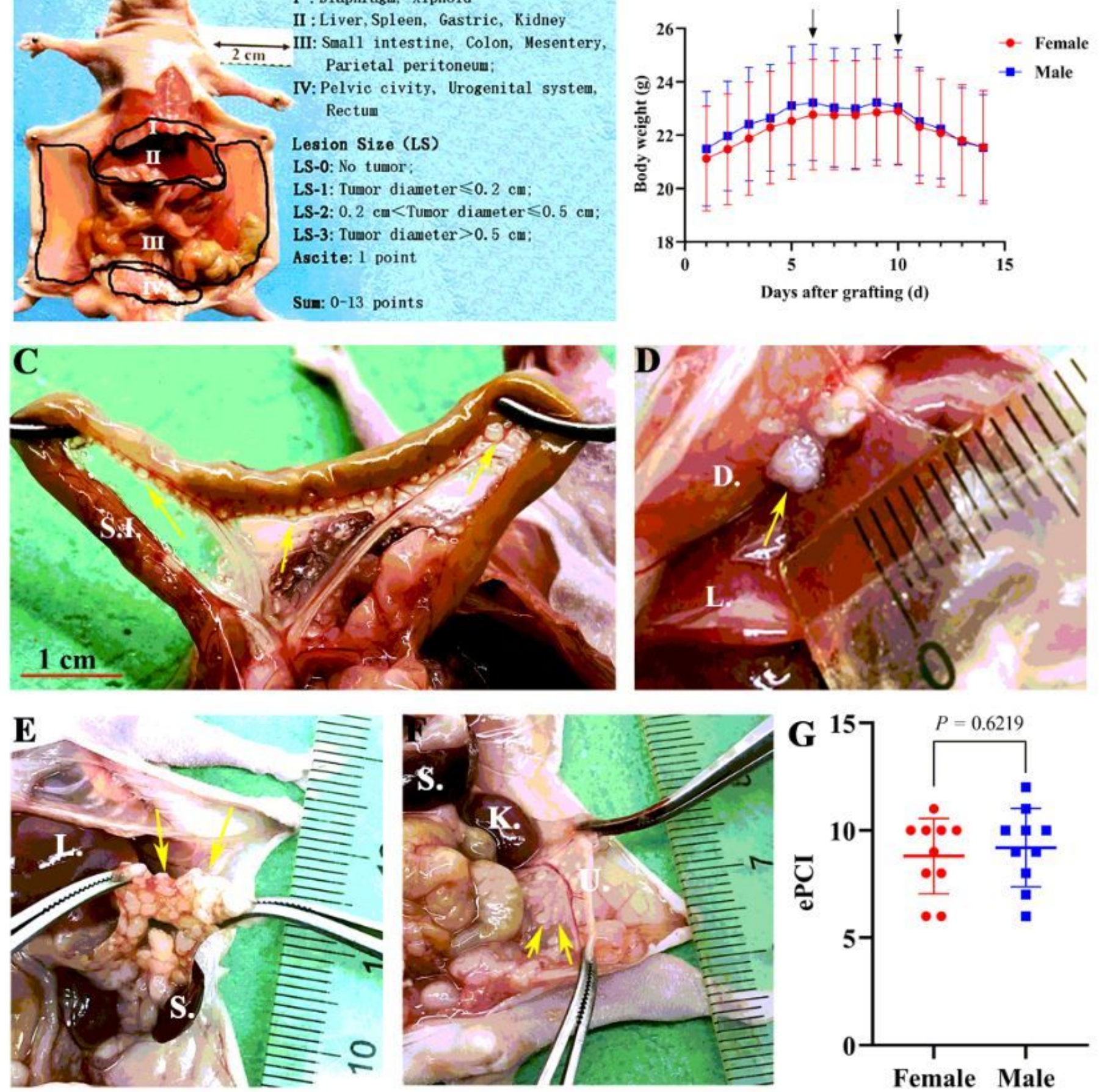

Figure 2

Experimental peritoneal cancer index (ePCl) score, body weight change, and the gross pathology of the new malignant peritoneal mesothelioma (MPM) patient-derived xenograft (PDX) models. A, The subarea and scoring of $\mathrm{ePCl}$ score system; $\mathrm{B}$, The body weight change of female and male nude mice; $\mathrm{C}$, The tumors invaded mesentery of the nude mice; D, Tumors invaded the diaphragm; $E$, Splenic mesentery of 
nude mice was invaded by tumors; F, The uterus of mice was invaded; G, ePCI score of female and male models. S.I., small intestine; D., diaphragm; L., liver; S., spleen; K., kidney; U., uterus.
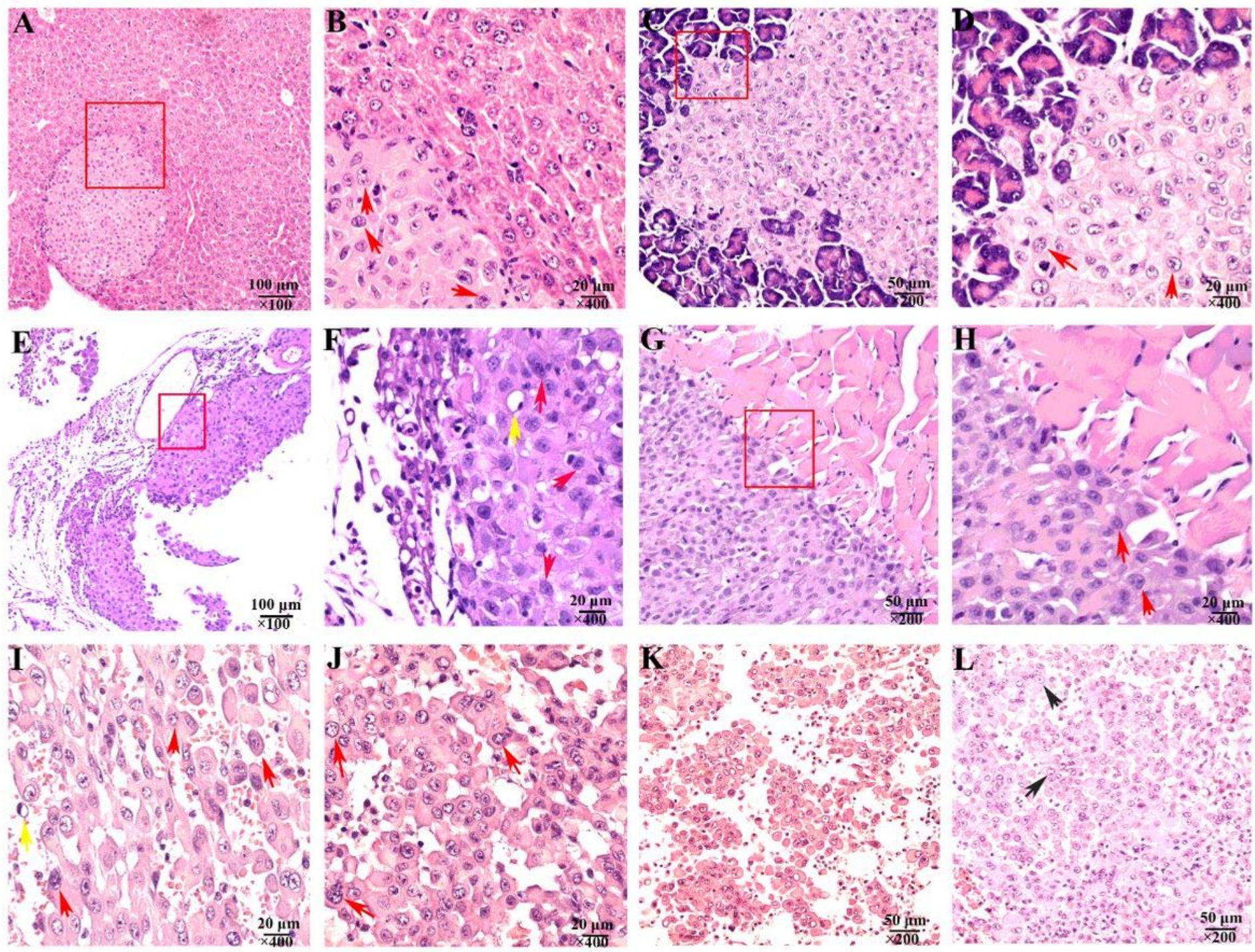

\section{Figure 3}

The Hematoxylin-eosin (HE) staining of the malignant peritoneal mesothelioma (MPM) patient-derived xenograft (PDX) models and the patient. $A \& B$, The tumors of models invaded the liver $(A, \times 100)$, the tumor cells were morphologically diverse, large in size, and markedly heteromorphic $(B, \times 400$; red arrow); $C \& D$, The tumors of models invaded the pancreas $(C, \times 200)$, and the tumor cells were markedly heteromorphic $(D, \times 400$; red arrow); $E \& F$, The tumors of models invaded the mesentery $(E, \times 100)$, the tumor cells were characterized by atypia (red arrow) and the signet ring cells ( $F, \times 400$; yellow arrow); $G \& H$, The tumors of models invaded the uterus $(G, \times 200)$, and the tumor cells were atypia $(H, \times 400$; red arrow); I\&J, The HE staining of the patient showed the tumor cells were also markedly heteromorphic (red arrow) and the signet ring cells were observed (I\&J, $\times 400$; yellow arrow); $\mathrm{K}$, The tumor cells of patient were arranged in a papillary structure $(K, \times 200)$; $L$, The tumor cells of patient were of clear cell type, and their cytoplasm was translucent $(L, \times 200$; black arrow). 

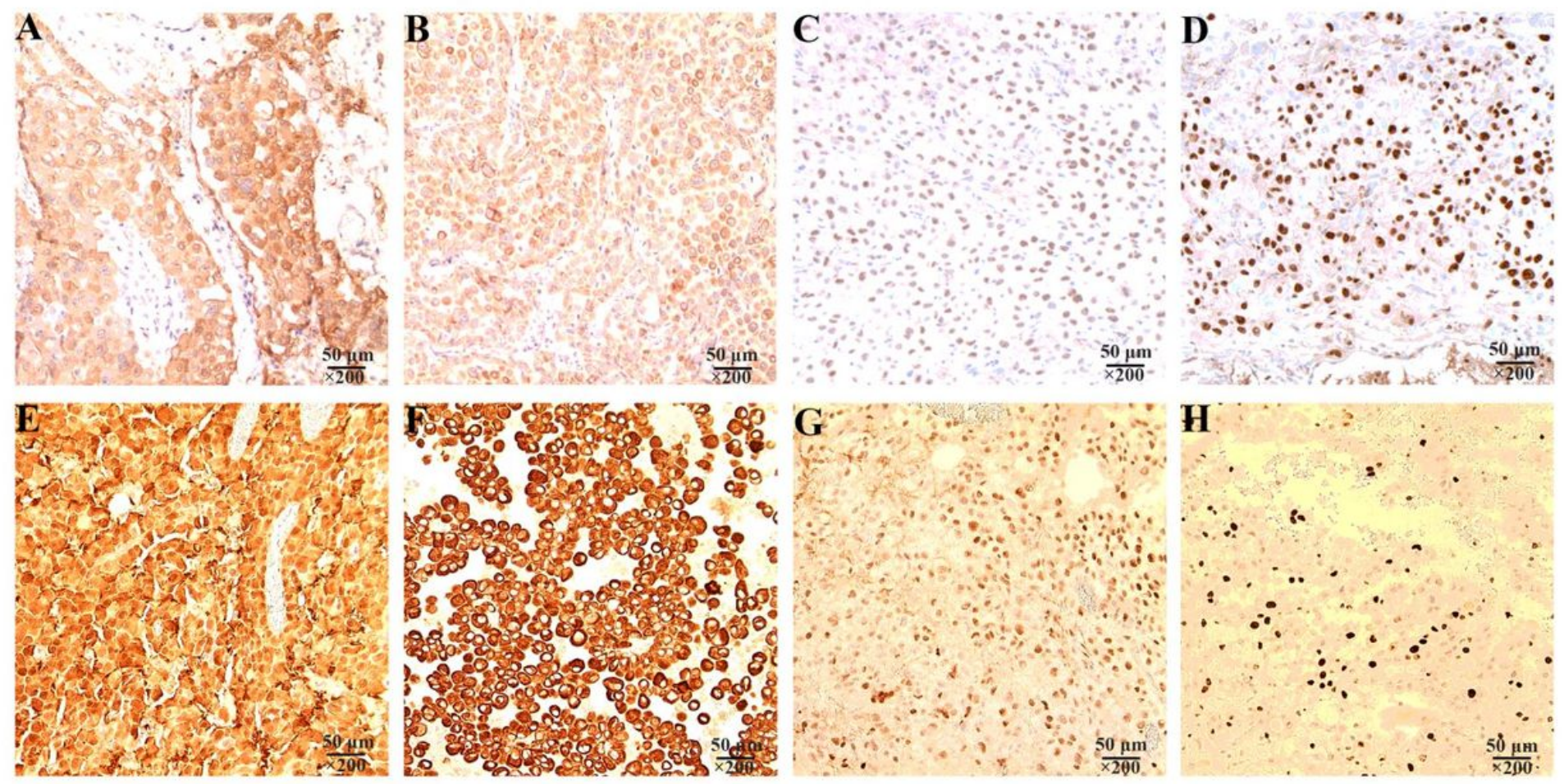

Figure 4

The immunohistochemical staining of the malignant peritoneal mesothelioma (MPM) patient-derived xenograft (PDX) models and the patient. A, The Calretinin of the tumor tissues of the models was positive, with the nucleus and cytoplasm were diffusely stained; $B$, The Cytokeratin $5 / 6$ of the models was positive; C, The WT-1 of the models was positive; D, The ki-67 of the models was positive, with the nucleus was stained; $E$, The Calretinin of the patient was positive; $F$, The Cytokeratin $5 / 6$ of the patient was positive; $G$, The WT-1 of the patient was positive; $H$, The ki-67 of the patient was positive, $(A-H, \times 200)$. 

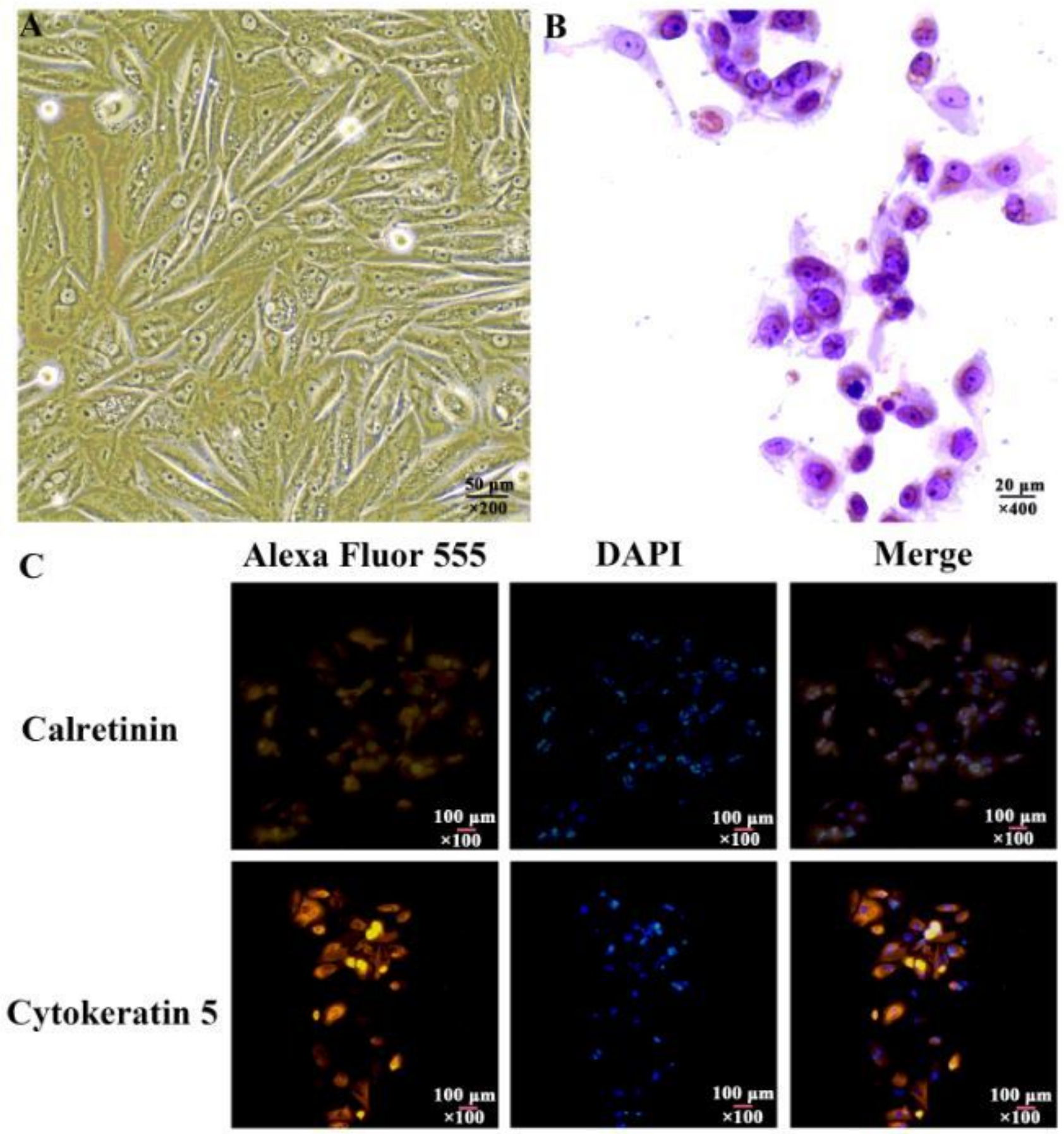

\section{Figure 5}

Cytological characteristics of primary malignant peritoneal mesothelioma (MPM) cell lines. A, MPM cells morphology was showed under the inverted phase contrast microscope $(A, \times 200)$; $B$, The diverse cell morphology was revealed by Swiss-Gimsa staining $(B, \times 400)$; $C$, The expression of Calretinin and Cytokeratin 5 were measured by immunofluorescence staining $(C, \times 100)$. 